\section{'I've started in the deep end'}

\author{
Emma Sunderland, from Manchester, UK, reflects on her graduation and \\ the early days of foundation training during the COVID-19 pandemic.
}

raduating by itself is no easy feat and with the addition of a global pandemic it appeared almost an impossible task. Before Monday 23 March, as the class of 2020 , we faced the usual challenges of final year: dental foundation training (DFT) interview, case patients, course work, competencies and revising for finals. When the UK-wide lockdown was announced we were, along with the rest of the nation, met with uncertainty and a rapidly changing environment. Our lives and future careers were paused. Would we be able to complete our examinations? Would we be able to graduate? Would we even be able to practise as dentists given the tight restrictions around aerosol generating procedures (AGPs)? What future implications does the virus hold on our profession?

Safety was the biggest priority. The COVID-19 infection rates grew exponentially in the early weeks and very quickly our universities had to adapt. The University of Manchester Dental Hospital was immediately closed, lectures done at a distance and examinations as we knew them completely changed. Frustration more than anything was the feeling amongst students. At the time questions swamped our tutors who were equally frustrated with the persistently developing and changing guidance.

Lockdown was the new challenge. I, like many others, found it difficult to motivate myself when all that was around me was unsettled. 2020 became the year of online video calls. We were still able to use remote access learning tools, participate in online lectures and revision sessions, but most importantly, we were able to contact our friends, families and colleagues at a safe distance to keep us motivated whilst still revising for final exams.

Fortunately, my preceding years of hard work were not lost. Along with most of my class at the University of Manchester, I managed to successfully complete all examinations and achieve a BDS degree which I had worked so hard for and I had

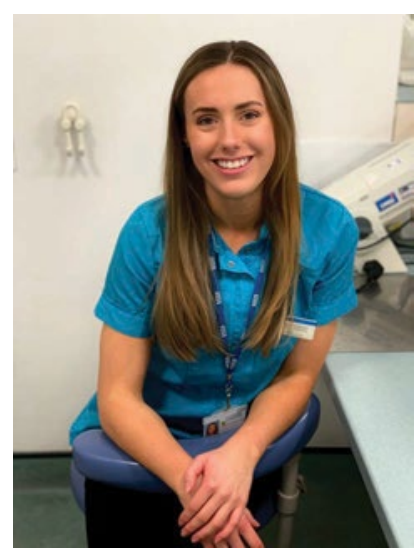

secured a training post at a practice for the following year. I did it! I finally was a newly qualified dentist. However, with the UK still in lockdown, parties and celebrations were placed on hold. The years of growing connections and friendships through the five years of dental school meant I was not allowed to enjoy the moment and throw my graduation cap with my friends which I had dreamt about.

Starting foundation training in such an unprecedented time presented me with new obstacles. After having faced the uncertainty with graduating, the challenge now with all the restrictions is meeting the targets set out by the deaneries.

I was anxious to begin foundation training. My last patient contact was March 2020 and I had not held a dental instrument since. Finally, I met my trainer and to my relief I quickly realised I was placed in a fantastic, supportive practice. Pressures of UDA targets for my supervisor, fully booked lists and hectic waiting rooms were diminished due to COVID-19. My educational supervisor made plenty of time to help me settle into the practice and find my feet.

Being a foundation dentist, I was expecting to be faced with adversity along the way. I hadn't imagined it would be quite so challenging. 'Emergencies only' was the advice and guidance at the time. I've started in the deep end, adapting to a new way of working with the PPE required for AGPs. Loupes paired with a stealth mask may possibly be one of the most challenging skills to master. Seeing complex patients needing urgent care definitely was a steep learning curve. We had to quickly learn how to triage over the phone, completely rethinking our training on antibiotics as we give out AAA advice. Having spent the last five years being taught and conveying to patients that antibiotics cannot cure toothache we find ourselves occasionally with our hands tied having only this in our armoury when operating at a distance.

Every practice is currently operating very differently depending on ventilation, air filtration systems and capacity. Each foundation dentist is encountering completely different clinical experiences from each other. This has led to a lot of pressure and anxiety when clinical targets have been announced. It is concerning that we may be unable to achieve such a wide range of clinical opportunities as our predecessors. I just hope this will not limit us in completing the training year and moving forwards with our careers.

Unlike in previous years, my study days are now held online. This first came with some appeal, no early commute, the comfort of the sofa and no late evenings. It hasn't been as idyllic as I once thought. Technical difficulties were met in the first few weeks as we all got used to this new way of learning. Glitchy microphones and resonance over the internet was harsh on the ears. Support networks and general socialising with others in our scheme have been at an all-time low in comparison to previous years. Learning and development is much harder with the limited, to almost absent, practical days I have had so far. I do worry whether I will be prepared and still be considered competent when I go for future jobs.

On the other hand, I have seen the profession develop and evolve to adapt to the 'new normal. I hope this too will happen with foundation dentists. I hope we are able to gain valuable experiences from this year and still drive forward in our professions. I am still ambitious and hope even during this time I go on to have a successful career in the future. I have confidence that in facing so many challenges at the very start of our careers we will become more resilient and versatile clinicians and I wish my fellow foundation dentists every success for the future. 